\title{
EI DUELO DE LOS PADRES: UNA MIRADA DESDE LA MASCULINIDAD
}

\author{
Mercedes Marqués Llompart ${ }^{1}$ \\ Carlos A. Rivera-Otero ${ }^{2}$
}

Recuerdo el día aquel en que con mucha emoción me dijiste: "Cuando yo sea grande ¡voy a ser como tú!”.

Escrito sobre la tumba de un pequeño niño, por su papá.

\section{Resumen}

Este artículo aborda la experiencia de la pérdida, específicamente la vivida por un padre ante la muerte de un hijo o hija. Los autores examinan las diferencias en la forma en que un padre y una madre se enfrentan al dolor de perder un hijo o hija y cómo estas diferencias pueden afectar su duelo y proceso de sanación. Asimismo cómo se afecta la relación de pareja y los hijos e hijas sobrevivientes. Esto se mira desde la socialización y los roles impuestos y aprendidos por género; y se analiza la construcción social de la masculinidad. Finalmente se discuten las implicaciones para el trabajo social.

Descriptores: pena, duelo, la pena de un padre, pérdida de un hijo o hija, construcción social de la masculinidad, masculinidades, masculinidad hegemónica.

\section{Abstract}

This article addresses the experience of loss; specifically, the one lived by a father upon the death of a son or daughter. The authors examine the differences in the way a father and a mother face the pain of losing a son or daughter and how this affects their mourning and healing processes. Also,

\footnotetext{
${ }^{1}$ Profesora en el Departamento de Trabajo Social, Facultad de Ciencias Sociales, Universidad de Puerto Rico en Río Piedras.

${ }^{2}$ Estudiante del Programa de Maestría, Escuela Graduada de Trabajo Social Beatriz Lassalle, Universidad de Puerto Rico en Río Piedras.
} 
how it affects their relationship as a couple, and their surviving children. This is being approached from the concept of socialization and the imposed and learned gender roles; and it analyzes the social construction of masculinity. Finally the authors discuss the implications for the profession of social work.

Keywords: grief, mourning, father's grief, parental loss of a child, social construction of masculinity, masculinities, hegemonic masculinity.

\section{Introducción}

La muerte de un hijo o una hija - a cualquier edad y por cualquier causa - es el dolor más intenso, desgarrador, agónico, incapacitante, abrumador, sobrecogedor de todos. No hay preparación ni solución posibles, desafía el orden natural del vivir y el morir; "un hijo no puede morir"... (Roccatagliata, 2000). Se le ha llamado 'la tragedia última' (Sarnoff Schiff, 1977), 'la pérdida última' (Marx \& Wenderhoff Davidson, 2005), que sólo se puede comparar o superar con la muerte de otro hijo o hija, algo inimaginable. "Primero te mueres"... (Levine, 2004), y entonces se inicia la dolorosa jornada de aprender a vivir otra vez; en un mundo que cambió para siempre, es un antes, es un después.

Una de las tareas en este volver a la vida es elaborar el duelo. Para esto hay que enfrentar la pena o aflicción, y hacerlo en la manera y al paso de cada cual. En la sociedad tecnológica que vivimos, que rinde culto a la belleza, la juventud, la salud, al estar vivo y funcional, no acepta como en el pasado la naturalidad de la muerte. Negación, miedo, rechazo a confrontarla e intolerancia ante los procesos 'lentos' del luto parecen imperar en la mayoría de las circunstancias individuales de muerte. Esta aparente insensibilidad no se manifiesta en tragedias masivas, aunque sí una prisa por la recuperación y normalización de la cotidianidad. El poder lidiar con un dolor tan profundo y continuar hacia la sanación, se dificulta por la falta de apoyo social donde los dolientes carecen de comprensión y compasión hacia su duelo y terminan aislados y revictimizados. Como la sociedad enfatiza la competencia, adecuación, fortaleza y logros, muchas veces son los familiares y amigos quienes evitan la expresión de sentimientos (Freeman, 2005; Golden, 2000; Kastenbaum, 2001; Kubler-Ross, 1969; Marx \& Wengerhoff Davidson, 2005; Mitchell et al, 2005; Rando, 1986; Rosenblatt, 2000a,b; Sarnoff Schiff, 1977, Simos, 1979). Esto se torna más difícil y complicado al interior de la 
familia y sobretodo de la pareja, cuando papá y mamá además tienen que enfrentar el que cada uno y una procese la muerte del hijo o hija de una manera diferente, sintiéndose desprovistos del apoyo del otro. También se extiende a los abuelos y abuelas, quienes sufren una doble aflicción, por su nieto o nieta fallecida y por el dolor de su hijo o hija en duelo y que quieren seguir protegiendo como cuando eran pequeños. Al abuelo no se le valida su dolor ni se espera que intervenga sin que se le solicite expresamente. En esta etapa de su vida lo aprendido de niño está muy bien internalizado y probablemente sienta que no debe llorar ni lamentarse o buscar apoyo (Gerner, 2004). Los hijos e hijas sobrevivientes, llamados los 'dolientes olvidados' pueden llegar a afectarse profundamente (ver Roccatagliata, 2003).

\section{La Construcción de la Masculinidad}

En la búsqueda de explicaciones al manejo de la pérdida de un hijo o hija por parte del padre surge la posibilidad de realizar un análisis desde la construcción social de la masculinidad. Esto como una de las posibles explicaciones, ciertamente no la única. La masculinidad es un discurso siempre cambiante de cómo ser hombre, cuyo rasgo más destacado es que equipara la masculinidad con la agresividad, la violencia, la competencia y la heterosexualidad. Este discurso es asumido por los hombres como un atributo central de su identidad. La masculinidad es un elemento constitutivo de los privilegios asociados a los hombres. Aunque no todos los hombres los comparten, hay factores que influencian el acceso a estos privilegios como pueden ser la raza, la clase social $u$ orientación sexual entre otros. La masculinidad no es un fenómeno uniforme, estático e igualmente compartido por todos los hombres (Román Tirado et al., 2003). Muchos autores identifican varios tipos de masculinidad o representaciones de la identidad masculina (Connell, 1995; Ramírez y García Toro, 2002). De aquí que se pluralice el término a masculinidades.

Badinter (1993) expresa que al hablar de identidades masculinas se afirma la idea que no existe una esencia natural de lo masculino. No hay una masculinidad única, lo que implica que no existe un modelo masculino universal y válido para cualquier lugar, época, clase social, edad, raza, orientación sexual. Más bien existe una diversidad heterogénea de identidades masculinas y de maneras de ser hombre en nuestra sociedad. Al respecto Román Tirado et al. (2003) argumentan que la masculinidad es un fenómeno cultural y no biológico. 
Ramírez (1999), expresa que lo masculino y lo femenino no constituyen una realidad separada del sujeto, son para este autor una construcción cultural cuyo fundamento no es biológico, esto a pesar de tener como base las diferencias biológicas. Es pues construido, diseñado, acordado y sostenido por un sistema de creencias, adscripciones y expectativas. Para Lomas (2004), la masculinidad es una construcción social en la medida en que a los hombres se les asigna socialmente unas u otras ideologías y tareas que influyen en las diversas y heterogéneas maneras de ser y sentirse hombres en nuestras sociedades.

La versión dominante de la masculinidad no constituye una esencia, sino una ideología de poder y de opresión que tiende a justificar la dominación masculina (Badinter, 1993). Connell (1997), se refiere a esta versión dominante como masculinidad hegemónica definiéndola así: "la configuración de práctica genérica que encarna la respuesta corrientemente aceptada al problema de la legitimidad del patriarcado, la que garantiza (o se toma para garantizar) la posición dominante de los hombres y la subordinación de las mujeres" (p.39).

La masculinidad hegemónica no es fija, ni es la misma en todas las sociedades ni a través de los tiempos. Es la masculinidad que ocupa la posición hegemónica en el sistema de relaciones de género dominante en determinado momento histórico. Esta masculinidad se convierte en norma y se ensalza y se destaca. Bourdieu (2000) señala que es aquella masculinidad que se expone como la forma natural de lo masculino y se estructuran dispositivos para legitimar su dominación.

\section{El modelo tradicional masculino}

Existe una imagen de lo masculino que ha sido transmitida de generación en generación. Argumenta Corsi (1995), que rara vez esta imagen relativa a la masculinidad se somete a una reflexión critica. Desde muy temprano en la infancia, los niños deben aprender lo que es ser 'un verdadero hombrecito'. Deben mostrarse fuertes, seguros de sí mismos, competitivos y ganadores. Este modelo de masculinidad incluye una serie de prohibiciones como: no llorar, no mostrarse débil, temeroso, inseguro entre otras características que podríamos seguir enumerando. Para muchos autores este modelo o imagen ideal es cercana a la omnipotencia. 
El modelo tradicional masculino, se construye sobre dos procesos psicológicos concurrentes y complementarios. El hiperdesarrollo del yo exterior manifestado en acciones dirigidas a hacer, lograr, actuar, en fin, ser un ganador. El segundo proceso es la represión de la esfera emocional. Este proceso consiste en no expresar los sentimientos propios. La expresión emocional es vedada, prohibida. Muchos hombres evitan cualquier situación de intimidad emocional y se niegan a pedir y aceptar ayuda. Para lograr un equilibrio entre estos dos procesos el hombre necesita ejercer un autocontrol para regular la expresión de sus emociones y sentimientos. Sentimientos que pueden ir desde el dolor, la tristeza, el placer, el temor, entre otros (Corsi, 1995).

Las dos características de este modelo masculino, planteado por Corsi (1995) se traducen en un estilo de relacionarse en los hombres caracterizados por la conducta afectiva y sexual restringida, actitudes basadas en modelos de control, poder y competencia y dificultades para el cuidado de la salud. La identidad masculina se construye evitando todo lo que es culturalmente asociado a lo femenino. Badinter (1993) expresa que la identidad masculina se construye por oposición, por un proceso de diferenciación de lo femenino.

Corsi (1995), indica que el miedo a lo femenino es el eje en el cual se va estructurando lo masculino y plantea una docena de mitos y creencias que sustentan la socialización masculina:

- La masculinidad es la forma más valorada de la identidad genérica.

- El poder, la dominación, la competencia y el control son esenciales como prueba de la masculinidad.

- La vulnerabilidad, los sentimientos y las emociones en el hombre son signos de feminidad, y deben de ser evitados.

- El autocontrol y el control sobre los otros y las otras y sobre su entorno son esenciales para que el hombre se sienta seguro.

- Un hombre que pide ayuda o trata de apoyarse en otros y otras, muestra signos de debilidad, vulnerabilidad e incompetencia.

- El pensamiento racional y lógico del hombre es la forma superior de la inteligencia para enfocar cualquier problema.

- Las relaciones interpersonales que se basen en emociones, sentimientos, intuiciones y contacto físico son consideradas femeninas y deben ser evitadas. 
- La intimidad con otros hombres debe ser evitada, porque les vuelve vulnerables y les pone en desventaja en la competencia por las mujeres o puede implicar homosexualidad.

- El éxito masculino en el trabajo y la profesión son indicadores de su masculinidad.

- La autoestima se apoya primariamente en los logros y los éxitos obtenidos en la vida laboral y económica (págs.16-17).

\section{El modelo masculino tradicional como un factor de riesgo para la salud integral de los hombres}

El tratar de sostener las exigencias de este modelo ha sido asociado como un factor de riesgo adverso a la salud y las estadísticas así lo confirman. En estadísticas de los Estados Unidos, los hombres encabezan el primer lugar en las primeras diez causas de muerte y presentan conductas de riesgo en mayor proporción que las mujeres según el Centro para el control de Enfermedades en el año 2000. Addis \& Mahalik (2003) argumentan que los hombres no buscan ayuda profesional en situaciones en los que la salud mental se ve afectada con la misma frecuencia que lo hacen las mujeres. "The Mens Health Network" (2003) indica que los hombres presentan tasas de mortalidad excesivas, su expectativa de vida es menor a la de las mujeres y están menos dispuestos a buscar servicios médicos.

Con respecto a la masculinidad hegemónica, se debe destacar que puede ser un determinante en la manifestación de conductas y actitudes de los hombres en prácticas de salud física, mental y conductas de alto riesgo como las mencionadas anteriormente (Courtenay, 2000). En Puerto Rico los hombres presentan 1.5 mayor prevalencia de enfermedades coronarias, mayores índices de muerte por cáncer, diabetes e infección del VIH (CDC, 2005). Los hombres también presentan altos índices de mortalidad respecto a accidentes. En el año 2002, estos representaron aproximadamente 5 veces más muertes en accidentes no intencionales, 4 veces más muertes en accidentes de vehículos de motor y 17.5 veces relacionadas con armas de fuego (CDC, 2005).

Los hombres están sobre representados en las estadísticas de suicidio, conductas violentas, conductas temerarias, el uso y abuso de drogas y alcohol entre otras (Brooks, 2001). Se sospecha que la depresión en hombres debe ser mucho mayor de lo que se pensaba previamente y de lo que reflejan las 
estadísticas; ante la tendencia de los hombres a negar y esconder la depresión por considerarlo algo estúpido o femenino (Brooks, 2001). Estos manifiestan su malestar a través de conductas que para ellos son socialmente más aceptadas, tales como el abuso de alcohol y la conducta agresiva.

De acuerdo a las estadísticas de la Policía de Puerto Rico, 91 por ciento de las personas que se suicidaron entre el año 2003 hasta mediados del 2007, fueron hombres. Puerto Rico, posee la media más alta de días en las que la salud física o mental de los hombres los mantuvo fuera del trabajo durante el mes previo al estudio, en todos los Estados Unidos (BRFSS, 2002). Los datos antes expuestos evidencian un deterioro en la salud física y mental de los hombres que de alguna manera pudiera estar relacionado con las exigencias de la masculinidad hegemónica según destaca Courtenay (2000).

Brooks (2001), conceptualiza la serie de conductas de alto riesgo en que se envuelven los hombres como el lado oscuro de la masculinidad. Los roles masculinos tradicionales producto del proceso de socialización crean un espectro amplio de patrones de conducta que hieren y lastiman profundamente a los hombres y que tienen sus consecuencias en la estructura social en la que vivimos. Entre las conductas de alto riesgo señaladas por Brooks (2001) se encuentra la violencia, el asalto sexual o violación, la promiscuidad sexual, el uso y abuso de alcohol y drogas, la depresión, el suicidio, paternidad poco comprometida o responsable entre otras.

\section{La pena por género, la pena de un padre}

La pérdida se ha definido como el haber sido privado de algo que se ha tenido y valorado o de alguien significativo. Provoca un cúmulo de sentimientos que a su vez definen la pena o aflicción. La pérdida de separación por muerte es una de las más importantes a través del curso de la vida de cualquier ser humano. Debido a razones de índole personal y culturales que tienden a evitar o excluir la pena, a muchas de estas personas enlutadas no se les permite experimentarla ni trabajarla saludablemente (Simos, 1979).

Muchos papás y mamás que han perdido un hijo o una hija experimentan problemas maritales significativos. La aflicción puede crear alejamiento y exacerbar problemas ya existentes. Esto le añade al luto una lucha paralela por mantener viva la relación de la pareja. De acuerdo con Rosenblatt $(2000 \mathrm{a}, \mathrm{b})$ algunos de estos problemas se relacionan con la comunicación, la 
sexualidad, la continuación del rol de padre y madre con los hijos e hijas sobrevivientes, el uso de drogas, alcohol, infidelidad, finanzas, culparse el uno al otro y sobretodo, las diferencias en el manejo (o no manejo) de la pena y el proceso de duelo y cómo estas diferencias son interpretadas. Bastantes autores del tema de la muerte de los hijos y el dolor de los padres y madres están de acuerdo con que la diferencia en el manejo de la pena y el duelo es el elemento más común e importante, y que las mismas están relacionadas a la socialización de roles y valores por género.

Betancourt Díaz (2007) relata las reacciones de padre y madre ante la muerte súbita de Ana María a los 5 años de edad, quien era hija única de una pareja muy sólida. Por esto también les sorprendió la forma diferente en que cada uno enfrentó su dolor. "Mientras Marisol lloraba y expresaba sus emociones, Víctor pensó que su responsabilidad era protegerla y evitar que llorara." Más adelante entendió que está bien expresar sus emociones. Roccatagliata (2000) expresa:

Con la muerte de un hijo se despiertan emociones muy fuertes y diferentes entre un hombre y una mujer. Por lo general, las mujeres expresamos nuestros sentimientos más abiertamente y lloramos en forma más frecuente. Necesitamos hablar y hablar de ese hijo que murió y de las circunstancias de su muerte. En cambio, los hombres inhiben el dolor y no muestran sus emociones, probablemente porque no fueron educados para eso. El hombre comparte lo que hace, no lo que siente. Muchos se vuelcan al trabajo para evitar acordarse de la muerte de su hijo, pero la angustia y desesperación la llevan por dentro. En nuestra cultura el hombre ha sido socializado para ser fuerte, autosuficiente y protector de la familia. La pérdida de un hijo representa para un padre la pérdida del control, lo despoja de su ego y del sentido de sí mismo, dejándolo sumido en un fuerte sentimiento de fracaso personal (pág.81).

Golden (2000) enfatiza que no hay soluciones fáciles ni prontas para la aflicción. Por lo que ante un problema sin solución la persona se va a sentir perdida, y la manera de enfrentarlo varía dependiendo del género. La mujer se refugia en la interacción con sus seres significativos; el hombre en la acción, por lo regular, solo. Schatz (2001) plantea que los roles 'típicos' de 
los hombres pueden interferir con la aflicción. Algunos de estos roles no son saludables porque no permiten expresar el verdadero ser y sentir. Entre éstos, según el autor, están el hombre macho, el competidor, el protector, el proveedor, el que soluciona problemas, el controlador y el hombre autosuficiente. El hombre macho nos recuerda aquello de "los hombres no lloran"; representa el siempre fuerte, no suavidad, debilidad, ni lágrimas. Contrario a la mujer, no puede hablar sobre su dolor. Petrie (2001) trae a su memoria la sentencia "Deja de llorar o te voy a dar algo para que llores de verdad" (p.43). A muchos hombres, dice, se les hace muy difícil llorar abiertamente, por eso se meten en su 'cueva' y sufren solos. Para él, su 'cueva' le ha ayudado, porque al menos ventila su pena. Krauss (1988), sostiene que un niño puede interpretar el llanto de su padre como una muestra de debilidad. El vivió esa experiencia con su hijo adolescente cuando tuvo que darle la triste nueva de la muerte de su mamá..."Lloré cuando le di la noticia y no pude dejar de llorar. Era la primera vez que él me veía llorar. Jeremy vio mi llanto como no varonil, me perdió el respeto y sintió que ya no podía contar conmigo para apoyo" (pág.134). No obstante, está bien visto y permitido que un padre llore todo el proceso de la muerte de su hijo, hasta su entierro. Otro de los roles, el de competidor, deja al padre derrotado; con la muerte no se puede competir ni mucho menos ganarle. El rol de protector es uno de los más intensos, pone sobre los hombros de papás y mamás una responsabilidad para toda la vida, pero también la sociedad mira hacia el padre como el protector principal. Giovanetti (2007), en una conmovedora historia sobre la muerte de su hijo, ofrece un ejemplo estremecedor sobre la renuncia instantánea - aunque temporera - de la expresión de su dolor al encontrarlo muerto en su propia cama, en su hogar, para que su esposa y sus otros hijos no vivieran allí lo que él estaba viviendo en ese momento.

Aunque mis emociones estaban tullidas y mi espíritu estaba quebrantado, mi intelecto y mi capacidad para reaccionar $\mathrm{y}$ tomar decisiones solamente flaquearon por unos instantes. Tomé rápidamente control de mi situación emocional, aunque ya la lanza había entrado hasta el tuétano de mis huesos y había perforado lo más profundo de mi corazón. Estaba desesperado, ansioso...mi alma estaba llorando aunque mis ojos no botaban lágrimas. No obstante, sabía que tenía que actuar y tomar decisiones...Lo más que me aterraba era [su esposa]. No me quería imaginar su reacción...Su nivel de emotividad y su apego a sus hijos auguraban una reacción 
explosiva al enterarse de la noticia. Tenía encima de mis hombros el dolor y angustia de la pérdida de un hijo, sumado a la preocupación de la reacción de mi familia...de mi esposa...Tenía que sacar a [su hijo] de la casa [a una sala de emergencia] sin que [ella] se enterara que estaba muerto...En mi mente solamente buscaba como disminuir el impacto en mi familia y asumí una posición de sobre protección ante el dolor de mi esposa y del resto de mis hijos (págs.40-41).

Otro testimonio, sorprendentemente muy parecido a éste, narra como este padre sacó de su casa, envuelta en sábanas a su hijita: "Cuando llegué...ella ya había muerto, y para no darle esa noticia a mi mujer la tomé en brazos y le dije que teníamos que llevarla a la clínica a pesar de que yo sabía que en mis brazos yacía mi hija muerta" (Roccatagliata, 2000, pág.109). El rol de protector se complica con sentimientos de culpa, una de ias emociones más fuertes ante una pérdida. Entre sus diversas manifestaciones, la culpa por no haberla evitado (Simos, 1979). Son los "si yo hubiera...", "si sólo yo...", "yo debí... (o no debí...), "no pude...". La sociedad puede ser muy cruel enjuiciando y exigiendo, sobretodo a los papás, a quienes le adjudican la responsabilidad de hacer, de ocuparse, de solucionar, de evitar: “¿y tú que hiciste?”, “¿y por qué no hiciste esto?”, “¿y por qué hiciste eso?”, “¿y por qué no hiciste nada?", "tú eras el responsable", "el hombre de la casa". Cuando un hijo muere, el padre siente que no lo protegió y el sentimiento de fracaso es bien grande; esto va atado a la culpa.

Como proveedor, debe regresar a su trabajo lo antes posible, sumergirse con extra intensidad en esta actividad le ayuda a no pensar. En cuanto a tener que solucionarlo todo, la muerte de un hijo no se puede resolver, pero quedan muchos otros problemas para los cuales ya no se siente capaz; tampoco ya no puede mantener el hogar y la familia bajo su control y finalmente ese hombre a quien le enseñaron la autosuficiencia, piensa que tiene que automanejar su duelo (Chethik, 2001; Levang, 1988; Petrie, 2001; Rando, 1986; Schatz, 2001). El condicionamiento social determina lo aceptable y lo conflictivo de la expresión de la pena. Esto deja al padre sin poder lidiar efectivamente con la tristeza, la depresión, la soledad. Le obstaculiza la expresión de emociones y sentimientos, el buscar ayuda profesional y apoyo de sus significativos (Rando, 1986; Hipple, 2007).

El siguiente testimonio de un papá con luto ejemplifica estos roles y sus efectos en la vivencia de la enfermedad y muerte de su hija: 
Nunca he vuelto a hablar [de ella]...a nombrarla. ¿Para qué meter el dedo en una herida que quiero que deje de sangrar?...Prefiero recordar en silencio...no voy a llorar, porque tenía que aparecer ante mi mujer íntegro y fuerte...trabajar duro [para] proveer adecuadamente...tenia que hacerme cargo...educado para asumir responsabilidades y no para dejarme vencer por el dolor...contener el llanto...sobrecarga la aflicción de los que me rodeaban...resistir...férreo...me educaron para sobrevivir. El saxo[saxofón] es el que llora...(Roccatagliata, 2000, pág. 105 )

El sentido de omnipotencia choca de frente con el de impotencia cuando muere un hijo. Ritter (2004), recordando el suicidio de su hijo, piensa en los niños pequeños que les gusta saltar desde lo alto, "cógeme Papi"...y tú los coges... ¿Pero y si llega un día en que ellos saltan y tú no puedes cogerlos? ...Yo tampoco pude agarrar a Bill. Pero...él no me dijo que iba a saltar. Tampoco esperó que yo pusiera mis brazos en posición. Pensando en esto...lloré y lloré." (pág.34).

\section{Implicaciones para la Práctica del Trabajo Social}

No importa en que escenario de práctica profesional se desempeñe, todo trabajador y trabajadora social, seguramente se enfrentará con alguna experiencia de muerte. La primera línea de ayuda en la gran mayoría de las instituciones sociales proviene de profesionales de trabajo social. Sus reacciones influirán en la comprensión, motivación y habilidad del doliente para trabajar su aflicción. Asimismo sus intervenciones contribuirán a que este proceso sea saludable. El dolor y la aflicción de experimentar una tragedia de tal magnitud puede causar trauma a nuestro ser físico, cognitivo, emocional, espiritual. La pena es un proceso individual y pertenece a cada cual. No obstante, la forma en que reaccionamos, procesamos y sobrevivimos depende de una multiplicidad de factores y circunstancias. La trabajadora y trabajador social necesitan estar bien adiestrados y preparados, no sólo en conocimientos sino adentro de sí mismos para poder enfrentarse a estas situaciones de pérdida. Por otro lado, estos profesionales de ayuda han sido socializados y son parte de la misma sociedad y tienen que hacer una introspección honesta de sus valores y conceptuaciones para no ser un elemento que obstaculiza sino que promueve. Miller y Golden (1998 a,b) invitan a mirar cara a cara al dolor de un padre con un espíritu inquisitivo, 
buscando entender, comprender, y aprender. Qué puede enseñar sobre la forma diferente y única en que pena. Asimismo que hay de positivo, saludable, efectivo y válido en ésta; a la que Golden (2000), le llama un don.

En el ejercicio de la práctica del Trabajo Social, es indispensable revisar, mirar y repasar los cimientos que dieron base a la creación y desarrollo de nuestra profesión. Sobre todo aquel principio que nos remite a luchar por acercarnos a la justicia social. Existe poca literatura escrita a la que tengamos acceso sobre la dimensión y condición masculina. Repasando la investigación social en esta área podemos ver como afecta el desarrollo y expone a los hombres a asumir conductas de alto riesgo y no poder disfrutar de una vida plena. Sin embargo en Puerto Rico, los esfuerzos de la práctica de la profesión dirigidos a concienciar a los hombres sobre la construcción social de la masculinidad, son escasos.

El enfoque primordial de este artículo, el manejo de la pérdida de un hijo o una hija se reviste de una importancia mayor porque requiere enfrentarse con uno de los atributos centrales con los cuales se construye socialmente la masculinidad, la represión emocional. La pérdida de un hijo o hija requiere la expresión de sentimientos, es experimentar el dolor, sentirse vulnerable, tal cual lo destacan Marx \& Wengerhoff Davidson, (2005), es la pérdida última. ¿Cómo puede un hombre entrenado desde niño para ser ganador experimentar semejante pérdida? El acercamiento que realizamos desde el construccionismo social, abre un sinnúmero de posibilidades para el análisis y las explicaciones. Esto debido a que da particular importancia a las subjetividades. Todo lo construido social y culturalmente, puede ser deconstruido y reconstruido. Los padres pueden aprender nuevas forma para manejar su dolor y sus aflicciones si así lo desean. Sobre todo aquel dolor ante la pérdida de un hijo o una hija. Por medio de este abordaje la profesión puede impactar diferentes contextos. Estos contextos pueden ir desde lo personal, lo social, lo cultural y lo político.

Neimeyer (2002), indica que el construccionismo social ve a los seres humanos como constructores de significados. El autor esboza seis preposiciones compatibles con la postura constructivista ofreciendo un punto de vista sobre la mortalidad y el duelo.

- La muerte como acontecimiento puede validar o invalidar las construcciones que orientan nuestras vidas o pueden constituir 
una nueva experiencia a la que no podamos aplicar ninguna de nuestras construcciones.

- El duelo es un proceso personal caracterizado por la idiosincrasia, intimidad, inextricabilidad de nuestra identidad.

- El duelo es algo que nosotros mismos hacemos, no algo que se nos ha hecho.

- El duelo nos da la oportunidad de reafirmar o reconstruir un mundo personal de significados que ha sido cuestionado por la pérdida.

- Cada sentimiento cumple una función, y debe entenderse como un indicador de los resultados de los esfuerzos que hacemos para elaborar nuestro mundo de significados tras el cuestionamiento de nuestras construcciones.

- Construimos y reconstruimos nuestras identidades como supervivientes a la pérdida negociando con los demás (pp.124136).

Según el autor la elaboración de duelo es un proceso que se da dentro de tres sistemas interdependientes e interrelacionados: el intrapersonal, el familiar y el social.

La profesión de Trabajo Social, debería revisar las implicaciones al bienestar de los hombres de la construcción social de la masculinidad y desarrollar práctica clínica con la diversidad de complicaciones que enfrentan los hombres. Este proceso en diferentes ámbitos debe llevar de acuerdo a Brooks (2001), a redefinir los roles del género masculino. Que los hombres no se valoren exclusivamente por su capacidad de proveer económicamente y proteger. Se le debe brindar mayor énfasis a la capacidad que tienen los padres para nutrir emocionalmente y brindar afecto. Si las expectativas sociales se focalizaran en la capacidad del hombre (padre) como nutridor emocional, el apego, la intimidad y la conexión emocional serian centrales en la masculinidad como lo son en la construcción social de la feminidad.

El cambio en la definición cultural de los roles de género, reconstruirá las relaciones familiares y generará nuevas ideas de cómo los hombres y las mujeres se comportan. Brooks (2001), señala como condición necesaria para la transformación en los roles de género, un cambio en la división sexual del trabajo en el hogar. Los niños y las niñas deberían ser socializados para desarrollar una variedad de características personales orientadas a expandir más que a restringir las posibilidades de género. Brooks (2001) también hace recomendaciones a terapeutas que trabajan con las diversas identidades 
masculinas. Entre éstas se encuentran la revisión personal del terapeuta de las reacciones que tiene hacia los estilos de conducta masculina, sobre todo aquellas catalogadas socialmente como negativas. Asumir que el participante masculino se encuentra en una situación de dolor, entendiendo que el modelo de masculinidad tradicional tiene beneficios y efectos dañinos. Contextualizar los problemas del cliente masculino desde la construcción social del género. El proceso terapéutico debe ser sensible a los aspectos culturales, incluyendo el género como un aspecto integral de la intervención, observando las situaciones del participante con relación a su contexto social, en el momento histórico determinado, transmitir empatía y entendimiento.

El ocultar el dolor emocional, es el secreto vergonzoso del hombre tradicional. Brooks (2001) indica que es muy necesario que los y las terapeutas reconozcan y validen el dolor y pánico psíquico de sus participantes varones. Todo este proceso debe llevar a un apoderamiento del hombre. De acuerdo con Golden (2000), el lado masculino en el proceso de sanar, no es tan aceptado como el más tradicional de expresión verbal y emocional de la pena; siendo este último más relacionado con el modo femenino. Esto de llorar y hablarlo con otras personas es considerado lo correcto (Chethik, 2001). Sin embargo dice Golden (2000), "el dolor de un hombre no se puede juzgar por apariencias externas o la abundancia de lágrimas" (pág.148). Su modo es más callado, menos visible, más conectado al futuro y a la acción (Golden, 2000); enfatizando el pensar, el actuar y el control emocional (Chethik, 2001).

La educación en Trabajo Social, debe crear más cursos sobre el manejo de la pérdida en todos los aspectos, sobre las masculinidades y las paternidades. Es muy necesario el desarrollo de modelos de intervención dirigidos a brindar alternativas a esta población. Estos modelos deben estar de acuerdo con las particularidades culturales y sociales, en nuestro caso, dentro de la realidad puertorriqueña. Concurrimos con Golden (2000) cuando afirma que todos y todas somos únicos en nuestras formas de penar y de buscar y encontrar la sanación, y que posiblemente estas diferencias individuales sean más que las diferencias por género. No obstante, enfatiza que estas últimas "sí existen y necesitan ser reconocidas" (pág.148). Kastenbaum (citado en Chethik, 2001), se pregunta si debemos sorprendernos de que el tema más descuidado sea la psiquis masculina. Finalmente, entendemos que es necesario promover la realización de investigaciones sobre el tema de la pérdida por muerte de un hijo o hija y sus efectos en la familia, para que puedan adelantarse nuevos 
conocimientos, teorías emergentes y modelos para la práctica profesional que permitan la inclusión de todas las formas de duelo y éstas sean validadas.

\section{Referencias}

Addis, Michael E. \& Mahalik, James R. (2003). Men, masculinity and the context of help seeking. American Psychologist, 58(1), 5-14.

Badinter, Elizabeth. (1993). XY: La identidad masculina. Madrid: Alianza.

Behavioral Risk Factor Surveillance System (2002). Recuperado el 6 de diciembre de 2007, desde www.cdc.gov/brfss.

Betancourt Díaz, Elba. (2007, 27 de agosto). Unidos ante la gran pérdida. $E l$ Nuevo Día, págs. 8-9.

Bourdieu, Pierre. (2000). La dominación masculina. Barcelona: Anagrama.

Brooks, Gary R. (2001). Masculinity and men's health. Journal of American College Health, 49(6), 285-297.

CDC. (2005).Healthy women: State trends in health and morality. Atlanta, GA: Autor.

Chethik, Neil. (2001). Father loss. New York: Hyperion.

Connell, Robert W. (1995). Masculinities. Berkley and Los Angeles: University of California Press.

Connell, Robert W. (1997). Gender \& power. California: Stanford University Press.

Corsi, Jorge. (1995). Violencia masculina en la pareja: Una aproximación al diagnóstico y a los modelos de intervención. Buenos Aires: Paidós.

Corsi, Jorge; Dohmen, Monica; Sotes, Miguel A. \& Bonino, Luis. (2004). Violencia masculina en la pareja: Una aproximación al diagnóstico y a los modelos de intervención (5ta edición). Buenos Aires: Paidós. 
Courtenay, W. (2000). Constructions of masculinity and their influence of men's well being: A theory of gender health. Social Science \& Medicine, 50, 1385-1401.

Estadísticas de Suicidios en Puerto Rico: Evolución anual por edad y género. Accedido el 10 de noviembre de 2007 desde www.tendeciaspr.com

Freeman, Stephen J. (2005). Grief \& loss: Understanding the journey. Belmont, CA: Thomson Brooks/Cole.

Gerner, Margaret H. (2004). For bereaved grandparents. Omaha, NE: Centering.

Golden, Thomas R. (2000). Swallowed by a snake: The gift of the masculine side of healing $\left(2^{\text {nd }}\right.$. ed.). Gaitherbird, MA: Golden Healing Publishing.

García Toro, Víctor I.; Ramírez, Rafael \& Solano Castillo, Luis (2004). Paternidades en Puerto Rico. Identidades. 2(2), 45-68.

Hipple, Eric. (2007). Real men do cry. In Healing the grieving heart (internet radio show hosted by Horsley, Gloria \& Horsley, Heidi). Accedido desde www.health.voiceamerica.com

Kastenbaum, Robert. (2001). Death, society, and the human experience. Rockleigh, NJ: Allyn \& Bacon.

Krauss, Pesach \& Goldfischer, Morrie (1988). Why me? Coping with grief, loss and change. New York: Bantam Books.

Kubler-Ross, Elizabeth. (1969). On death and dying. New York: MacMillan

Levang, Elizabeth. (1998). When men grieve. Minneapolis, MIN: Fairview Press

Levine, Marie (2004). First you die. Learn to live after the death of your child. New York: Silver Thread Publishers. 
Lomas, Carlos (2004). Los Chicos también lloran: Identidades masculinas, igualdad entre los sexos y coeducación. Barcelona, España: Paidós

Marx, Robert \& Wengerhoff Davidson, Susan (2005). Facing the ultimate loss: Coping with the death of a child. Fredonia, WI: Champion Press

Miller, James E. \& Golden, Thomas R. (1998a). A man you know is grieving. Wayne, IN: Willowgreen Publishing.

Miller, James E. \& Golden, Thomas R. (1998b). When a man faces grief. Wayne, IN: Willowgreen Publishing.

Mitchell, Ellen \& Barkin, Carol; Cohen Audrey; Colleti, Lorenza; Eisenberg, Barbara; Goldstein, Barbara J. et al. (2005). Beyond tears: Living after losing a child. New York: St. Martin's Press.

Neimeyer, Robert A. (2002). Aprender de la pérdida. Una guía para afrontar el duelo. Buenos Aires: Paidós.

Petrie, Ronald G. (2001). Into the cave: When men grieve. Portland, Oregon: One to Another Publisher.

Ramírez, Rafael (1999). Dime capitán: Reflexiones sobre la masculinidad (2da ed.). Río Piedras: Ediciones Huracán.

Ramírez, Rafael, García-Toro, Víctor \& Cunnigham, Ineke (Eds.). (2002). Caribbean masculinities: Working papers. San Juan, Puerto Rico: HIV/AIDS Research and Education Center.

Rando, Therese A. (Ed.) (1986). Parental loss of a child. Champaign, IL: Research Press.

Ritter, William A. (2004). Take the dimness of my soul away. New York: Morehouse Publishing.

Roccatagliata, Susana (2003). La otra cara del dolor. Hijos que pierden hermanos. Santiago de Chile: Grijalbo.

Roccatagliata, Susana (2000). Un hijo no puede morir. La experiencia de seguir viviendo. Santiago de Chile: Grijalbo. 
Roman Tirado, Felix; González Armenteros Juan; Fernández Bauzó, Edwin; Cruz Díaz, Edwin \& Ávila Rodríguez, Marviliz (2003). Masculino que ninguno: una perspectiva sociopersonal de género, el poder y la violencia. San Juan, Puerto Rico: Colectivo Ideologías y Vivencias de los Géneros.

Rosenblatt, Paul C. (2000a) Help your marriage survive the death of a child. Philadelphia, PA: Temple University Press.

Rosenblatt, Paul C. (2000b). Parents grief: Narratives of loss and relationships. Philadelphia, PA: Brunnel/Mazel.

Sarnoff Schiff, Harriet. (1977). The bereaved parent. New York: Penguin Books.

Schatz, William H. (2001). Healing a father's grief. Issaquah, WA: Medic Publishing.

Simos, Bertha G. (1979). A time to grieve: Loss as a universal human experience. New York: Family Service Association of America.

The Men's Health Network. (2003). The silent health crisis. The Men's Health Network. Accedido el 6 de diciembre de 2007, desde http://www.mesnhealthnetwork.org/library/silenthealthcrisis2003a.pdf 